randomised trial designed to study the effects of low dose glucocorticoids (GC) in elderly patients with Rheumatoid Arthritis (RA). The research team is also committed to promoting a better understanding of the risks and benefits of these drugs among health professionals and patients. In order to achieve these goals, it is important to assess the current concepts and concerns of health professionals (HP) regarding GCs.

Objectives: In this study, we evaluated the beliefs about GC benefits and risks of HP who regularly use and monitor them in the treatment of RA.

Methods: These surveys were disseminated to HP who have experience dealing with RA patients in their daily clinical practice. These surveys mainly enrolled physicians, but also nurses or physiotherapists who considered themselves experienced in this field. The surveys were made available in Portuguese and English, and disseminated through the GLORIA investigational team. National medical societies contributed by inviting clinicians to participate.

Regarding the questions on GC efficacy, HP could signal (dis)agreement on a 5 point scale: disagree, slightly disagree, neutral, slightly agree, and agree. Agreement was defined as the proportion of HP answering slightly agree or agree. Survey Monkey software was used to disseminate the online surveys.

Results: Responses provided by $130 \mathrm{HP}$ are summarised in table 1. The results are presented taking all participating countries in account as the rates are similar between them. Most responses came from The Netherlands $(57 \%)$ and Portugal (34\%). Almost all participants were physicians (97\%).

Efficacy of GC was highly endorsed. Close to $90 \%$ of HP considered low dose GC were very effective in the control of signs and symptoms of RA, and agreed that GC improve RA symptoms within days. Almost $80 \%$ agreed that GC reduce structural damage, and $85 \%$ disagreed that GC lose their efficacy after a few months.

The opinions of health professionals regarding frequency of GC adverse events are presented in table 2.

Regarding GC AE events, most of the respondents considered that low dose GC adverse events were very rare or rather rare, except for glycaemic control in patients with diabetes and osteoporosis. Acne and cardiovascular events were evaluated as very rare adverse events by approximately one third of HPs. However, there was significant heterogeneity in the responses.

Abstract AB1410HPR - Table 1. Health professional's characteristics $(n=130)$ and data on GC's efficacy.

\begin{tabular}{l|c|} 
Country of participants, number (\%) & $70(57)$ \\
Netherlands & $41(33)$ \\
Portugal & $11(9)$ \\
Others & $122(97)$ \\
Physicians, number (\%) & \\
Level of agreement for statements, number (\%) & \\
At a dose $\leq 7.5$ mg prednisolone/day, GC: & $88(90)$ \\
$-\quad$ are very effective in the control of signs and symptoms of RA \\
$\quad$ improve RA symptoms within days & $86(88)$ \\
GC reduce the probability of articular damage caused by RA & $77(79)$ \\
In RA these doses of GC lose efficacy after a few & $15(15)$ \\
$-\quad$ months of therapy & $18(18)$ \\
- years of therapy &
\end{tabular}
health professionals who attended this survey (\% of HP per frequency category).

\begin{tabular}{l|c|c|c|c|c|c|c|} 
& None & $\begin{array}{c}\text { Very } \\
\text { rare } \\
(<1 \%)\end{array}$ & $\begin{array}{c}\text { Rather } \\
\text { rare } \\
(1-3 \%)\end{array}$ & $\begin{array}{c}\text { Rather } \\
\text { frequent } \\
(4-10 \%)\end{array}$ & $\begin{array}{c}\text { Frequent } \\
(11-20 \%)\end{array}$ & $\begin{array}{c}\text { Very } \\
\text { frequent } \\
(>21 \%)\end{array}$ & $\begin{array}{c}\text { Don't } \\
\text { know }\end{array}$ \\
Acne & 13 & $\mathbf{3 3}$ & 29 & 19 & 2 & 1 & 4 \\
Mood disturbances & 8 & 19 & $\mathbf{4 0}$ & 28 & 4 & 0 & 2 \\
Poor DM control & 1 & 6 & 15 & $\mathbf{4 2}$ & 27 & 9 & 1 \\
$\begin{array}{l}\text { Cardiovascular } \\
\text { events }\end{array}$ & 6 & $\mathbf{3 4}$ & 33 & 16 & 3 & 0 & 9 \\
$\begin{array}{l}\text { Osteoporosis } \\
\text { Serious infections }\end{array}$ & 1 & 7 & 21 & $\mathbf{4 3}$ & 19 & 7 & 3 \\
& 13 & 33 & $\mathbf{4 2}$ & 11 & 1 & 0 & 1
\end{tabular}

Conclusions: GC are widely used drugs in RA. The vast majority of participating HPs are convinced that GCs are efficacious in the treatment of $R A$, including DMARD effects, and retain this efficacy long term. However, concerns about severe side-effects are also very prevalent.

Acknowledgements: Funding: This project has received funding from the European Union's Horizon 2020 research and innovation programme under grant agreement No 634886

Disclosure of Interest: None declared
DOI: 10.1136/annrheumdis-2018-eular.6920

\section{AB1411-HPR HYPERMOBILITY AND FIBROMYALGIA: COEXISTENCE OR MISTAKEN IDENTITY?}

P Donohue $^{1}$, S. Chavrimootoo ${ }^{2}$, S. Ramakrishnan ${ }^{2} .{ }^{1}$ Department Of Physiotherapy, ${ }^{2}$ Department of Rheumatology, Our Lady's Hospital, Navan, Meath, Ireland

Background: The 2010 classification of fibromyalgia (FM) has many overlapping features with hypermobility-Ehler Danlos Syndrome (hEDS) such as chronic widespread musculoskeletal pain, sleep disturbances, fatigue and anxiety. ${ }^{2,4,5}$ Indeed hEDS may be misdiagnosed as FM because of diffuse pain with a strong myofascial component. ${ }^{1}$ Chopra ${ }^{1}$ believes these should be considered as two distinct conditions which may co-exist. By classifying as one and ignoring the other are we doing our clients a disservice?

The five point questionnaire $(5 P Q)$ is a simple questionnaire used to detect hypermobility and is claimed to have good reproducibility in addition to satisfactory sensitivity and specificity. ${ }^{3}$ This audit examined the numbers of FM clients that also fulfilled the hEDS criteria according to the $5 P Q$

Methods: 66 clients with a consultant diagnosis of FM who attended the multidisciplinary FM education session were invited to fill in the $5 \mathrm{PQ}$ along with their fibromyalgia impact questionnaire (FIQ). 57 of the 66 completed both questionnaires over a 13 month period.

The cut off points of 2 out of 5 for the $5 \mathrm{PQ}$ and 50 on the FIQ were used.

The higher numbers on the $5 P Q$ indicate increased likelihood of hypermobility.

Around 50 on the FIQ is typical for FM with higher values indicating increased severity

The individual scores for the FIQ question on anxiety were also examined. Question 19 rates anxiety on a visual analogue scale from $0-10$, the higher values indicating increased levels of anxiety.

Results: 54 females and 3 males aged between 18 and 76, mean age 51

15 of the $57(26 \%)$ scored 2 or above on the $5 \mathrm{PQ}$ suggestive of hypermobility.

Of those $15,10(66 \%)$ scored greater than 50 on the FIQ thus meeting the criteria of FM also.

7 of the $15(47 \%)$ scored 8 or above on the anxiety scale.

Conclusions: From this audit $26 \%$ of FM clients also scored positively for hEDS as per the 5PQ. hEDS may account for some or all of thei FM symptoms.

The $5 P Q$ is a quick and easy tool to use. It may improve hEDS detec tion rates and could be used in a busy rheumatology clinic.

Further research is required to ascertain whether those with positive scores meet the physical criteria for hEDS.

\section{REFERENCES}

[1] Chopra P, Tinkle B, Hammonet C, Brock I, Gompel A, Bulbena A, Francomano C, 2017. Pain management in the Ehlers-Danlos Syndromes. Am. J Med. Genet. PartC Semin. Med Genet. 175C:212-219.

[2] Hakim AJ, Keer R, Grahame R, 2010.Hypermobility, Fibromyalgia and Chronic Pain. Churchill Livingstone Elsevier.

[3] Hakim AJ, Grahame R, 2003. A simple questionnaire to detect hypermobility: an adjunct to the assessment of patients with diffuse musculoskeletal pain. Int. J. Clin. Pract. 57:163-166, 2003b.

[4] Tinkle B, Castori M, Berglund B, Choen H, Grahame R, Kazkaz H, Levy H. 2017 Hypermobile Ehlers-Danlos Syndrome (a.k.a Ehlers-Danlos Syndrome Type III and Ehlers-Danlos Syndrome Hypermobility Type): Clinical Description and Natural History. Am J Med Genet Part C Semin Med Genet 175 C48-69.

[5] Wolfe F, Clauw DJ, Fitzcharles M, Goldenberg DL, Katz RS, Mease P, Russel AS, Russel IJ, Winfield JB, Yumus MB. 2010 The American College of Rheumatology Preliminary Diagnostic Criteria for Fibromyalgia and Measurement of Symptom Severity. Arthritis Care \& Research Vol. 62, No. 5, May 2010, pp 600-610.

Disclosure of Interest: None declared DOI: 10.1136/annrheumdis-2018-eular.2425 


\section{HPR Epidemiology and public health (including prevention)}

\section{AB1412-HPR IS DIFFUSE ALVEOLAR HAEMORRHAGE IN ANCA- ASSOCIATED VASCULITIS PREDICTIVE OF POOR PROGNOSIS?}

L. Garcia, C. Pena, R. Aguila Maldonado, A.C Costi, A. Testi, V. Nagua, M. A. Garcia. Rheumatology, Hospital San Martin La Plata, La Plata, Argentina

Background: Diffuse alveolar haemorrhage (DAH) is a serious complication of anti-neutrophil cytoplasmic antibody (ANCA)-associated vasculitis (AAV). Detailed characteristics of patients with AAV and associated DAH have been limited to case reports and a few case series, due to the rarity of the condition $\left(.^{1}\right.$ Prompt diagnosis is required as early treatment is crucial.

Objectives: To compare clinical manifestations, laboratory and inmunological values, morbidity and mortality in patients with ANCA-associated vasculitis (AAV) with and without Diffuse Alveolar haemorrhage (DAH)

Methods: Retrospective cohort study. Data from the medical records of patients over the age of 18 were evaluated between the years 20002017. Ninety patients with diagnosis of AAV who met the criteria of ACR 1990 Classification or Chapel Hill Concensus Conference 2012 were included. DAH was diagnosed based on minor or major hemoptysis and/ or respiratory insufficiency together with at least 1 positive result on $x$-ray and/or computed tomography scan. The sample was divided in two groups: with DAH (group 1) and without DAH (group 2) along the disease. We compared demographic data, Birmingham Vasculitis Activity Score (BVAS) and Five Factor Score (FFS) at the onset of the desease, hemogram, creatinine and ANCA antibodies by inmunofluoresence and/or ELISA, clinical manifestations as renal, neurological, mucocutaneous, articular, cardiological, oftalmological and ear, nose and throat (ENT) damage and mortality between groups.

Chi-square or Fisher's exact test was used for dichotomous variables as appropriate. P-value $<0.05$ was considered statistically significant. Logistic regression analysis was used to identify predictors of survival.

Results: Group 1 included 24 patients (66\% male, mean age at onset of the disease 54 years). Most frequent type of vasculitis was Granulomatosis with polyangeitis (GPA) $54 \%$. Mean BVAS 21 points and FFS 1 point. Group 2 included 66 patients: $36 \%$ male, mean age of the desease 52 years, GPA 45\%, BVAS was 17 points and FFS de 0 points. Table 1 shows demographic, clinical data and mortality of each group. Logistic regression analysis showed statistically significant difference between DAH and male sex (p: 0.017 OR $6.08 \mathrm{Cl}$ 95\% $1.37-$ 26.92).

Abstract AB1412-HPR - Table 1

\begin{tabular}{|c|c|c|c|c|}
\hline & $\begin{array}{c}\text { With HAD n=24 } \\
(26.6 \%)\end{array}$ & $\begin{array}{c}\text { Without HAD n=66 } \\
(73.4 \%)\end{array}$ & $\mathrm{p}$-value & $\mathrm{OR}(\mathrm{Cl} 95 \%)$ \\
\hline Male sex & $16(66.7 \%)$ & $24(36.4 \%)$ & 0.011 & $\begin{array}{c}3.50(1.30- \\
9.38)\end{array}$ \\
\hline BVAS & 21 points & 17 points & 0.009 & \\
\hline FFS & 1 point & 0 point & $<0.001$ & \\
\hline Anaemia & $21(87.5 \%)$ & $30(45,5 \%)$ & $<0.001$ & $\begin{array}{c}8.4(2.28- \\
30.91)\end{array}$ \\
\hline $\begin{array}{l}\text { Mucocutaneous } \\
\text { disease }\end{array}$ & $1(4.2 \%)$ & 19 (28.8\%) & 0.018 & $\begin{array}{c}0.11(0.01- \\
0.91)\end{array}$ \\
\hline ENT disease & $7(29,2 \%)$ & 35 (53\%) & 0.045 & $\begin{array}{c}0.36(0.13- \\
0.99)\end{array}$ \\
\hline $\begin{array}{l}\text { Neurological } \\
\text { disease }\end{array}$ & $4(16.7 \%)$ & $31(47 \%)$ & 0.009 & $\begin{array}{c}0.22(0.07- \\
0.73)\end{array}$ \\
\hline Renal damage & $24(100 \%)$ & $31(47 \%)$ & $<0.001$ & $\begin{array}{c}1.70(1.40- \\
2.23)\end{array}$ \\
\hline RPGN & $13(54.2 \%)$ & $12(18.2 \%)$ & 0.001 & $\begin{array}{c}5.31(1.92- \\
14.71)\end{array}$ \\
\hline Dyalisis & $8(33.3 \%)$ & $4(6.1 \%)$ & 0.003 & $\begin{array}{c}6.8(1.69- \\
27.35)\end{array}$ \\
\hline Mortality & $5(22 \%)$ & $12(21 \%)$ & 0.930 & $\begin{array}{c}1.05(0.32- \\
3.44)\end{array}$ \\
\hline
\end{tabular}

Conclusions: DAH was associated with increased morbidity but not modified the mortality in this group of patients. The results seem to be agree on the Five Factor Score $^{2}$ that does not include DAH within parameters.
REFERENCES:

[1] West, S, et al. Diffuse Alveolar Haemorrhage in ANCA-associated Vasculitis. Intern Med 52: 5-13, 2013.

[2] Guillevin, L, et al. The Five- Factor Score revisited: assessment of prognoses of systemic necrotizing vasculitides based on the FVSG cohort. Medicine (Baltimore) 2011; 90: 19-27.

Disclosure of Interest: None declared

DOI: 10.1136/annrheumdis-2018-eular.5361

\section{AB1413-HPR ADVANTAGES OF RHEUMATOLOGY NURSING VACCINATION OF PATIENTS WITH INFLAMMATORY RHEUMATIC DISEASES OF AUTOIMMUNE ORIGIN. ANALYSIS OF THE FIRST 3 YEARS}

A Pons-Bas ${ }^{1}$, E. Ivars ${ }^{1}$, C. Cano ${ }^{1}$, M. Lorente ${ }^{1}$, J. Rosas ${ }^{1}$, G. Santos-Soler ${ }^{1}$, E. Salas ${ }^{1}$, J.M. Senabre-Gallego ${ }^{1}$, X. Barber ${ }^{2}$, on behalf of AIRE-MB Group. ${ }^{1}$ Rheumatology Department, Hospital Marina Baixa, Villajoyosa (Alicante); ${ }^{2} \mathrm{ClO}$, Miguel Hernández University, Elche, Spain

Background: Patients with systemic autoimmune diseases (SAD) have higher incidence of infections. In our Service, until 2014 about $50 \%$ of patients did not complete vaccination for pneumococcus or hepatitis B virus (HBV).

Objectives: To know the characteristics of vaccinated patients and advantages of vaccination by Rheumatology nurse (Nurse-RHEU).

Methods: Observational study of patients in follow-up in rheumatology, vaccinated from 2015-2017, by SAD in treatment with biological therapy and/or immunosuppressive, by Nurse-RHEU, following the recommendations from SVR and the Valencian Society of Preventive Medicine and Public Health Consensus Document for the vaccination of patients with SAD, published in 2014. Nurse-RHEU, assumed from 2015 the vaccination of all rheumatology patients with SAD. The vaccines to be administered were: pneumococcal (13 V and/or $23 \mathrm{~V})$ and hepatitis $B$ virus (HBV).

Pneumococcal vaccination should be initiated with the conjugate/13 V type and at least 8 weeks later with a dose of polysaccharide/23 $\mathrm{V}$ vaccine. A single $23 \mathrm{~V}$ booster dose is recommended 5 years after the first dose. Only if patient have received a previous dose of $23 \mathrm{~V}$, it is advisable to administer type $13 \mathrm{~V}$, one year later. For the HBV vaccine, it is necessary previously check the immune status of the subject. If it is not immune, 3 doses will be administered (0-1-6 months).

Since 2015, our Section has: refrigerator, electronic access to the Vaccinated Nominal Registry of the Valencian Community (it collects data of the patient, date, type, batch of vaccine administered), specific database (epidemiological data of the patients, future dose programming, access to electronic medical records) and Nurse-RHEU trained. Previously, the nurse details possible side effects, and how to act or contact.

Results: Of the 261 patients vaccinated during 2015 to $2017,65 \%$ were women, with mean age $53.57 \pm 15.50$ years (10-81 years). The diagnosis was: rheumatoid arthritis: $48 \%$, ankylosing spondylitis: $23 \%$, psoriatic arthritis: $13 \%$, systemic lupus erythematosus: $7 \%$, uveitis: $4 \%$, and others $5 \%$. The reason for vaccination was: initiation treatment with a biological drug $(51 \%)$, or immunosuppressive $(49 \%)$. A total of 621 vaccines were administered, which were: 13-valent conjugate antineumotococcal: 259 (42\%), 1 st VHB dose: 94 (15\%), 2nd VHB dose: 85 (14\%), 3rd VHB dose: 72 (12\%), pneumococcal 23-valent polysaccharide: 111 (17\%). All vaccines were registered in the Vaccinated Nominal Register of the Valencian Community.

Conclusions: 1. Patients with SAD in biological treatment and/or immunosuppressant, vaccinated by Nurse-RHEU, achieve a completeness of all doses of vaccination, close to $100 \%$. 2. In a large part of the patients, vaccination is scheduled at the beginning of the diagnosis of the disease, at the first consultation or immunosuppressive treatment, the same day of the visit to the Rheumatologist, avoiding unnecessary visits to the patient. 3. An adequate electronic registry allows immediate access to information from any point in the Valencian Community, through access to the computer program of the Health department.

Acknowledgements: The study was supported with a research grant from the Association for Research in Rheumatology of Marina Baixa (AIRE$\mathrm{MB})$.

Disclosure of Interest: None declared DOI: 10.1136/annrheumdis-2018-eular.4934 